\title{
Monte Carlo Sampling Based In-Home Location Tracking With Minimal RF Infrastructure Requirements
}

\author{
Gergely V. Záruba ${ }^{1}$, Manfred Huber ${ }^{1}$, Farhad A. Kamangar ${ }^{1}$, and Imrich Chlamtac ${ }^{2}$ \\ ${ }^{1}$ Department of Computer Science and Engineering, The University of Texas at Arlington \\ \{zaruba, huber, kamangar\}@uta.edu \\ ${ }^{2}$ Erik Jonson School of Engineering, The University of Texas at Dallas chlamtac@utdallas.edu
}

\begin{abstract}
This paper describes research towards a system for locating users in a home environment requiring only a minimal wireless infrastructure. The only sensor reading used for the location estimation is the radio frequency received signal strength indication (RSSI) measured by an RF Interface (e.g., Wi-Fi). Location estimates are computed using Bayesian filtering on sample sets derived by Monte Carlo sampling. Wireless signal strength maps for the filter are obtained by a two-step parametric and measurement driven ray-tracing approach to account for absorption and reflection characteristics of various obstacles. Our trace driven simulations indicate that RSSI readings from a single access point in an indoor environment are sufficient to derive good location estimates of users.
\end{abstract}

Keywords - localization, geo-positioning, RSSI, ray-tracing

\section{INTRODUCTION AND MOTIVATION}

Location-aware computing [2,10] is a recent research paradigm relying on the knowledge of the physical location of mobile users. Location awareness is also an important enabler of pervasive computing, where the more information is available on the physical environment of users and computing entities the better the applications on the network can adapt to the user. In this paper we restrict ourselves to in-door localization, more precisely to in-home localization (without loosing the generality to apply our results to any indoor environment). Our work relies only on received signal strength measurements from wireless radio access points to determine the location of users. The major contribution of our paper is to show that good results can be achieved with readings from a single access point. This is in contrast to previous works where at least three access points were required to localize users on the corridors (only) of office environments.

\section{A. Localization Techniques}

The best-known location determination system is the Global Positioning System (GPS) [7]. A GPS receiver can estimate its location by measuring the propagation time of radio signals from several satellites to the receiver. Although, after the recent liberalization of the GPS the precision of the obtained GPS position is quite accurate (down to a meter), many commercial GPS receivers need to have line-of-sight to at least three satellites in the sky. Although GPS is the most wide spread (and global) positioning system, there are several other approaches available for location estimation and even more have been proposed in the literature. The types of sensors used to obtain the location information vary from ultrasonic through photonic to radio signal strength measurement sensors. Since our work is focused towards the indoor environment we will not revisit other outdoor positioning systems. In the indoor environment, infrared and laser transmitter/receiver systems [23], ultrasonic sensor/actuator systems [16], computer vision systems [5,12], physical contact [15] and close proximity radio identification (RFID) sensor [22] based localization systems have been proposed and built to track mobile users.

Due to the fact that most mobile users that need to be tracked are enabled with wireless radio communication network interfaces today, such as Wi-Fi (IEEE802.11b), protocols that provide location estimates based on the received signal strength indication (RSSI) of wireless access points $[3,13,17,19,24]$ are becoming more-and-more precise and sophisticated. Yet, all of the above listed approaches need RSSI readings from at least 3 access points at each location to provide with some precision.

In general, RSSI based positioning includes two phases: i) the training phase where the wireless map of the environment is determined by field measurements and ii) the positioning phase where position calculation is performed based on the wireless map. In [14] the authors apply an extended Kalman filter [6] to RSSI measurements of cellular base-stations. Although [14] considers relatively macro-term outdoor movements, it was the first major work (to our best knowledge) to apply statistical methods to RSSI measurements for localization. RSSI based measurement techniques can be broadly divided into deterministic and probabilistic techniques:

Deterministic techniques include [1] and [19] where the location area is subdivided into smaller cells and readings are taken in these cells from several known access points (training phase). In the positioning phase the most likely cell is then selected, i.e., the cell that best fits the current measurement.

Probabilistic positioning techniques include [3, $6,13,17,24]$ where a probability distribution of the user's location is defined over the area of the movement. The goal of the positioning is to reach a single mode for this distribution, which is the most likely location of the tracked user. In [6] the authors establish and train a Bayesian belief network with a preset number of discretized location possibilities (cells). By inverting the Bayesian network, they derive the conditional probabilities of a user being at the different cells given the current RSSI reading. The results of this approach show a very coarse location determination with a large computational and memory overhead. In [24], the authors use a similar Bayesian model except that inversion calculations are not made for all base-stations, but for only the strongest subset of them Both [6] and [24] apply their systems to office hallways. The model in [17] is a generalized version of [3] that applies machine 
learning techniques to the Bayesian network to increase precision (which in turn increases the computational burden). In [13] the authors take the Bayesian approach a step further by including directions of users in their model.

Our work can be categorized as a probabilistic approach. The main tool and theme throughout the proposed work will be Bayesian filtering using Monte Carlo (MC) sampling (introduced in [5]), where the probability distribution of the location of users is captured, followed, and calculated by sampling. This method can use an arbitrary a-priori distribution converging (or "collapsing") to a single mode of the sampled distribution. This method is computationally less expensive than evaluating Bayesian networks.

\section{B. Wireless Maps- The Training Phase}

To estimate location from signal strength readings it is necessary to have a spatial statistical representation of the received signal strengths from the surrounding access points. All the above mentioned RSSI based indoor localization approaches rely on a long "training" phase where the entire target area is measured with some spatial precision. However, such data collection/measurement requires significant human labor. Often, it would be preferable to be able to use a simple model of the environment to determine a model for the signal strength's distribution. A number of such modeling techniques have been devised for managed wireless networks in office environments. In [8] the authors evaluate ray-tracing techniques that are used to derive indoor propagation models while in [9] a statistical approach is used that builds a wireless map based on statistical properties of rooms and the area.

\section{Sequential Monte Carlo Sampling}

Probabilistic approaches to mobile node localization from RSSI measurements rely on the precise estimation of a posterior probability distribution, $p\left(s_{t} \mid d_{1}, \ldots, d_{t}\right)$, of the likelihood of the node's state (location), $s_{t}$, given a history of the received measurements, $d_{1}, \ldots, d_{t}$. The goal is to derive a representation that deals with missing information about the motion of the mobile node and the uncertainty present in the measurement data. The main problem when using such probabilistic representations is that they can be prohibitively complex. As a result, most existing approaches to localization using RSSI measurements rely either on the discretization of space into a small number of regions of interest $[1,9,19,24]$ or an unrealistic model of the uncertainty of the measurements $[14,19]$. An example of the latter can be seen in Kalman filterbased approaches which have to make the assumption that the probability distribution for the locations as well as the measurement error model are Gaussian. However, in the presence of highly ambiguous measurements these distributions are generally multi-modal, indicating the existence of multiple possible positions that match a particular set of RSSI readings.

In recent years, MC sampling-based techniques for the estimation of probability distributions have been developed [4,5] and applied to different problems [11,5,21]. In these simulation-based techniques, empirical probability distributions, $p_{N}(s)$, are represented by a set of $N$ weighted, samples, $\left\{\left(s^{(i)}, w^{(i)}\right) \mid i \in[1, N]\right\}$ as $\quad p_{N}(s)=\sum_{i=1}^{N} w^{(i)} \delta_{s^{(i)}}(s)$, where $w^{(i)}$ is the weight of sample $s^{(i)}$ and $\delta_{s^{(i)}}(s)=\delta\left(s^{(i)}-s\right)$ is the Dirac delta function. This distribution approximates the actual probability distribution, $p(s)$, as

$$
\int_{s 1}^{s 2} p(s) d s \approx \int_{s 1}^{s 2} p_{N}(s) d s=\sum_{s^{(i)} \in[s 1, s 2]} w^{(i)}
$$

As a result, MC sampling-based techniques can be used to represent arbitrary probability distributions as long as the number of samples is sufficiently high. Calculations of posterior distributions $p(s \mid d)$ in these techniques are performed by re-sampling operations on the samples representing the prior distribution $p(s)$. The computational complexity of these MC techniques is therefore determined by the number of samples used to represent the distribution. Computation time can be traded off against precision by modifying the number of samples used.

\section{WIRELESS MAP CALCULATION}

This section outlines our five-step approach to derive wireless signal strength maps.

\section{A. Step One: Floor-Plan}

To obtain a wireless map, the first step is to define the environment. This requires measuring the wall, window and door lengths of rooms as well as identifying major obstacles. In our example we took the house of one of the authors, which was already equipped with a Wi-Fi access point. We have defined four different obstacle types: brick walls, interior walls, windows and doors. We have used the xfig format due to its simple design to define the floor-plan. The map is depicted in Figure 1, where black lines around the perimeter are brick walls $\left(\mathrm{O}_{1}\right)$, grey lines around the perimeter are windows $\left(\mathrm{O}_{2}\right)$, grey lines inside the house are interior walls $\left(\mathrm{O}_{3}\right)$ and dark lines inside the house are doors $\left(\mathrm{O}_{4}\right)$. The grey square indicates the location of the access point and the grey circles represent measurement points.

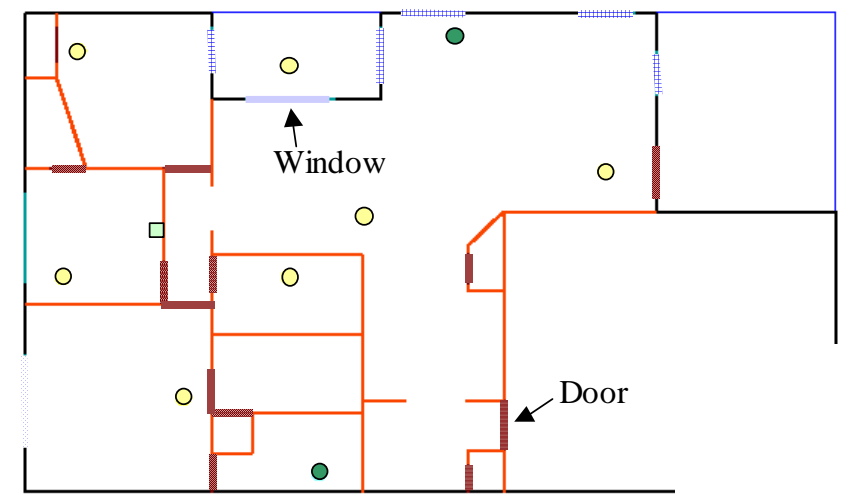

Figure 1. Floor-plan of a house with measurement

\section{B. Step Two: Measurement and Measurement Points}

In the second step the user is required to define measurement points well-spread across the floor-plan (as 
represented by the gray circles in Figure 1). The number of measurement points to be defined depends on the number of obstacles in the floor-plan. To obtain good results, at least twice as many measurement points are needed as there are obstacle types (as explained in the next subsection). In our example we have defined 10 measurement points ( 8 that are needed and an additional 2 for increased precision) since we have $b=4$ different obstacle types.

\section{Step Three: Parametric Ray-tracing}

In this step we are running a ray-tracing algorithm to find the signal strength of the access point at all the measurement points as a function of the transmission and reflection parameters of the obstacles. Wi-Fi transmits in the $2.4 \mathrm{GHz}$ band, where the radio signal coming off the access point can be approximated with the sum of single straight-line propagation rays emanating in all directions around the omni-directional antenna of the access point [8]. Each obstacle is assumed to reflect and transmit (let through) some portion of the energy of radio wave. $R_{O_{i}}$ and $T_{O_{i}}$ are defined as the reflection and transmission coefficients for the $\mathrm{i}^{\text {th }}$ obstacle type $(0<\mathrm{i} \leq \mathrm{b})$, where $\left(\mathrm{R}_{\mathrm{O}_{\mathrm{i}}}+\mathrm{T}_{\mathrm{O}_{\mathrm{i}}}\right)<1$. For example if $\mathrm{O}_{1}$ is in the way of a ray, then it is assumed to reflect $\mathrm{R}_{\mathrm{O}_{1}}$ portion while letting $\mathrm{T}_{\mathrm{O}_{1}}$ portion of the same incoming energy propagate through.

Rays are generated by the access point in all directions but to keep the process computationally feasible, the direction of the rays has to be discretized between $[0,2 \pi)$ with precision $\Delta \alpha$. The $\lfloor 2 \pi / \Delta \alpha\rfloor$ rays are then traced one-by-one sequentially. A ray hitting an obstacle will cease to exist but will spawn two other rays, one going on in the same direction (if the perimeter is not reached) while the other bouncing back from the obstacle. For each of the rays, the distance traveled, the number and type of obstacles reflected from and the number and type of obstacles transmitted by is stored, until the distance traveled reaches a pre-defined threshold. If a ray hits the area of a measurement point, then its current parameters (distance traveled, number and type of different transmissions and reflections) are stored for that measurement point. Let us denote the number of reflections from obstacles $\mathrm{O}_{1}$ to $\mathrm{O}_{b}$ by $\mathrm{r}_{1} \ldots r_{b}$, the number of transmissions by obstacles $\mathrm{O}_{1}$ to $\mathrm{O}_{b}$ by $t_{1} \ldots t_{b}$, and the distance of travel by $d$. The power of this ray at the measurement point is: $\frac{P_{0}}{d^{2}} \prod_{i=1}^{b} R_{O_{i}}^{r_{i}} * \prod_{i=1}^{b} T_{O_{i}}^{t_{i}}$. Let us denote the number of rays going through measurement points $M_{1} \ldots M_{m}$, by $\mathrm{N}_{\mathrm{M}_{1}} \ldots \mathrm{N}_{\mathrm{M}_{\mathrm{m}}}$ respectively. Thus for each measurement point:

$$
P_{M_{j}}=\sum_{k=1}^{N_{M_{j}}}\left(\frac{P_{0}}{{ }^{k} d^{2}} \prod_{i=1}^{b}{ }^{k} R_{O_{i}}^{r_{i}} * \prod_{i=1}^{b}{ }^{k} T_{O_{i}}^{t_{i}}\right)
$$

where $\mathrm{R}_{\mathrm{O}_{1}} \ldots \mathrm{R}_{\mathrm{O}_{\mathrm{b}}}$ and $\mathrm{T}_{\mathrm{O}_{1}} \ldots \mathrm{T}_{\mathrm{O}_{\mathrm{b}}}$ are unknown, and upper-left indices represent ray indexes for the $\mathrm{k}^{\text {th }}$ ray.

\section{Step Four: Obstacle Parameter Determination}

The signal strength measurements obtained in the second step can now be used as the values for $\mathrm{P}_{\mathrm{M}_{1}} \ldots \mathrm{P}_{\mathrm{M}_{\mathrm{m}}}$. By looking at Equation 1 we note that we have $2 b$ unknowns. In step-four we will estimate these unknowns finding values for the reflection and transmission rates of all obstacles. In order to solve the above polynomials for the $2 b$ unknowns, we need at least $m \geq 2 b$ equations, i.e., measurement points. Due to the coarse modeling of the environment, we have to also include an error term into the measurement. Let us denote the measured power levels by $\mathrm{W}_{\mathrm{M}_{1}} \ldots \mathrm{W}_{\mathrm{M}_{\mathrm{m}}}$. Then for all $i$, where $m \geq i \geq 1 ; \mathrm{W}_{\mathrm{M}_{\mathrm{i}}}=\mathrm{P}_{\mathrm{M}_{\mathrm{i}}}+\mathrm{e}_{\mathrm{i}}$. We can now calculate a least squares error estimate for all unknowns by minimizing function $F$ :

$$
F\left(R_{O_{1}} \ldots R_{O_{b}}, T_{O_{1}} \ldots T_{O_{b}}\right)=\sum_{j=1}^{m} e_{j}^{2}=\sum_{j=1}^{m}\left(W_{M_{j}}-P_{M_{j}}\right)^{2}
$$

Minimization of $F$ is a complex problem due to the number of variables, and the order of the individual polynomials. Thus, to minimize $F$ we employ a heuristic optimization technique We have chosen simulated annealing (SA) to obtain such sub-optimal values for $\mathrm{R}_{\mathrm{O}_{1}} \ldots \mathrm{R}_{\mathrm{O}_{\mathrm{b}}}$, $\mathrm{T}_{\mathrm{O}_{1}} \ldots \mathrm{T}_{\mathrm{O}_{\mathrm{b}}}$. Our SA algorithm takes the polynomial description file generated in the third step, and the measured values for the measurement points and $\mathrm{P}_{0}$ (received power at a one meter distance in a free propagation environment) as input and provides with the estimated values for the reflection and transmission parameters for all defined obstacles.

\section{E. Step Five: Ray-tracing for Wireless Power Maps}

We calculate the actual wireless power map in this step.. The first task is to define the angular precision of the raytracing as well as defining the resolution of the wireless power map by discretizing the target area into $\Delta$ s side-sized square cells. $\Delta$ s has little impact on the complexity of computation of the ray-tracing however it influences the precision of the location estimate. The ray-tracing process is similar to that of the third step with the exception that rays leave a scalar power level footprint in all cells they travel through. Figure 2 shows a visualization of the wireless power map for our sample floorplan, with $\Delta \mathrm{s}=0.3 \mathrm{~m}$.

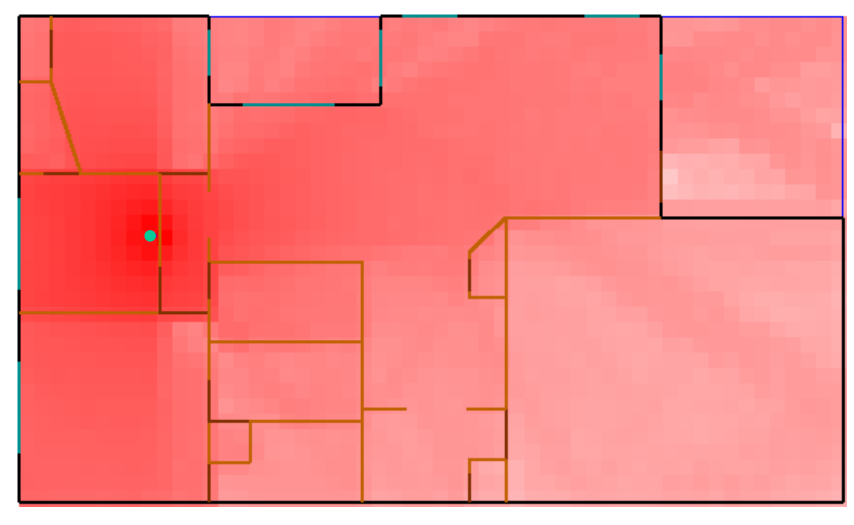

Figure 2. Calculated wireless map.

\section{MONTE CARLO SAMPLING-BASED BAYESIAN-FILTERING}

This section describes the basic approach to mobile node localization from RSSI measurements and introduces three estimation models that have been implemented and tested. The 
goal is to obtain an estimate of the posterior probability distribution, $p\left(s_{t} \mid d_{1}, \ldots, d_{t}\right)$, of potential states, $s_{t}$, using the RSSI measurements, $d_{1}, . ., d_{t}$, and the wireless power map introduced in the Section II. The calculation of the distribution is performed recursively using a Bayes filter:

$$
p\left(s_{t} \mid d_{1}, \ldots, d_{t}\right)=\frac{p\left(d_{t} \mid s_{t}, d_{1}, \ldots, d_{t-1}\right) \cdot p\left(s_{t} \mid d_{1}, \ldots, d_{t-1}\right)}{p\left(d_{t} \mid d_{1}, \ldots, d_{t-1}\right)}
$$

Assuming that the Markov assumption holds, i.e., $p\left(s_{t} \mid s_{t-1}, \ldots, s_{0}, d_{t-1}, \ldots, d_{1}\right)=p\left(s_{t} \mid s_{t-1}\right)$, the filtering equation can be transformed into the recursive form:

$$
p\left(s_{t} \mid d_{1}, \ldots, d_{t}\right)=\frac{p\left(d_{t} \mid s_{t}\right) \cdot \int p\left(s_{t} \mid s_{t-1}\right) \cdot p\left(s_{t-1} \mid d_{1}, \ldots, d_{t-1}\right) d s_{t-1}}{p\left(d_{t} \mid d_{1}, \ldots, d_{t-1}\right)},
$$

where $p\left(d_{t} \mid d_{1}, \ldots, d_{t-1}\right)$ is a normalization constant. In the case of the localization of a mobile node from RSSI measurements, the Markov assumption requires that the state contains all available information that could assist in predicting the next state. Thus, an estimate of the non-random motion parameters of the node is required as part of the state description. Starting with an initial, prior probability distribution, $p\left(s_{0}\right)$, a system model, $p\left(s_{t} \mid s_{t-1}\right)$, representing the motion of the mobile node, and the measurement model, $p(d \mid s)$, it is then possible to derive new estimates of the probability distribution over time, integrating one new measurement at a time. Each recursive update of the filter can be broken into two stages:

Prediction: Use the system model to predict the state distribution based on previous readings

$$
p\left(s_{t} \mid d_{1}, \ldots, d_{t-1}\right)=\int p\left(s_{t} \mid s_{t-1}\right) \cdot p\left(s_{t-1} \mid d_{1}, \ldots, d_{t-1}\right) d s_{t-1}
$$
estimate

Update: Use the measurement model to update the

$$
p\left(s_{t} \mid d_{1}, \ldots, d_{t}\right)=\frac{p\left(d_{t} \mid s_{t}\right)}{p\left(d_{t} \mid d_{1}, \ldots, d_{t-1}\right)} p\left(s_{t} \mid d_{1}, \ldots, d_{t-1}\right)
$$

To address the complexity of the integration step and the problem of representing and updating a probability function defined on a continuous state space, the approach presented uses a sequential Monte Carlo filter to perform Bayesian filtering on a sample representation. As introduced in Section I.C, a distribution is represented by a set of weighted random samples and all filtering steps are performed using Monte Carlo sampling operations. In particular, the initial sample distribution, $p_{N}\left(s_{0}\right)$, is represented by a set of uniformly distributed samples with equal weights, $\left\{\left(s_{0}^{(i)}, w_{0}^{(i)}\right) \mid i \in[1, N], w_{0}^{(i)}=1 / N\right\}$, and the filtering steps are performed as follows:

Prediction: For each sample, $\left(s_{t-1}^{(i)}, w_{t-1}^{(i)}\right)$, in the sample set, randomly generate a replacement sample according to the system model, $p\left(s_{t} \mid s_{t-1}\right)$. This result in a new set of samples corresponding to $p\left(s_{t} \mid d_{1}, \ldots, d_{t}\right)$

$$
\left\{\left(\tilde{s}_{t}^{(i)}, w_{t}^{(i)}\right) \mid i \in[1, N], w_{t}^{(i)}=1 / N\right\}
$$

Update: For each sample, $\left(\tilde{s}_{t}^{(i)}, w_{t}^{(i)}\right)$, set the importance weight to the measurement probability of the actual measurement, $\widetilde{w}_{t}^{(i)}=p\left(d_{t} \mid \tilde{s}_{t}^{(i)}\right)$. Normalize the weights such that $\sum_{i \in[1, N]} \eta \cdot \widetilde{w}_{t}^{(i)}=1.0$ and draw $N$ random samples for the sample set $\left\{\left(\widetilde{s}_{t}^{(i)}, \eta \cdot \widetilde{w}_{t}^{(i)}\right) \mid i \in[1, N]\right\} \quad$ according to the normalized weight distribution. Set the weights of the new samples to $1 / N$, resulting in a new set of samples $\left\{\left(s_{t}^{(i)}, w_{t}^{(i)}\right) \mid i \in[1, N], w_{t}^{(i)}=1 / N\right\} \quad$ corresponding to the posterior distribution $p\left(s_{t} \mid d_{1}, \ldots, d_{t}\right)$.

To apply the filter to the problem of mobile node localization from RSSI measurements a measurement model has to be provided. Three different motion models were tested:

\section{A. Simple Sequential Monte Carlo Filter}

The simplest localization algorithm uses a system model assuming that at every point in time, the node moves with a random velocity drawn from a Normal distribution $N(0,1)$ (in metric units). No information about the environment is included in this model, and as a consequence, the filter permits the estimates to move along arbitrary paths. Since this motion model does not consider any past motions, the state of the localization filter can be represented as a vector of the $\mathrm{x}$ and $\mathrm{y}$ location, $s=\left(\begin{array}{ll}x & y\end{array}\right)^{T}$.

\section{B. Simple Sequential MC Filter with Boundary Information}

To model the effects of boundaries and limit the simulated sample trajectories to physically possible ones, the second filter uses information about the location of the walls to modify the Gaussian velocity model by limiting available choices to that do not lead to collisions. Figure 3 shows an example for this motion model, which corresponds to a random displacement limited by the walls. The figure shows the probability density of moving to a new location within one step assuming that the mobile node is located at the center of the distribution. In the same way as in the filter in Section A, this filter uses a two-dimensional state vector, $s=\left(\begin{array}{ll}x & y\end{array}\right)^{T}$.

\section{Sequential MC Filter with Internal Motion Model}

Due to the inertia of a physical node moving in the environment, the random displacement model used in Section A might not be sufficiently realistic. The third movement model assumes that nodes tend to move at a constant velocity. Thus the model relies on the previous velocity with a current random acceleration, where the acceleration is drawn randomly from a Normal distribution $a \sim N\left(0 \mathrm{~m} / \mathrm{s}^{2}, 1 \mathrm{~m} / \mathrm{s}^{2}\right)$. To permit the use of this model, the state used in this filters has to include an estimate of the current velocity of the mobile node and is thus a four-dimensional vector consisting of the position and velocity of the mobile node, $s=\left(\begin{array}{llll}x & y & v_{x} & v_{y}\end{array}\right)^{T}$. To address walls, samples that collide with walls in the prediction step of the filtering update are assigned a weight of 0 .

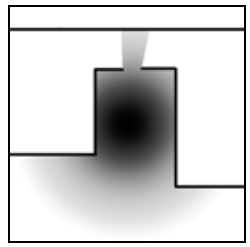

Figure 3. Probabilistic Displacement Model with Wall Limitations 


\section{PERformanCE STUDIES}

The goals of our evaluation studies are: i) to validate our ray-tracing based wireless power map with real-life measurements, and ii) to simulate a scenario where a mobile user is tracked using our filtering methods.

\section{A. Wireless Map Calculation Studies}

Our ray-tracing map generation was run with an angular precision of $\Delta \alpha=2 \pi / 360$ and square cells of size $0.3 \mathrm{mx} 0.3 \mathrm{~m}$ covering the floor-plan. We have taken and averaged 50 measurements for each of the 10 measurement points.

To evaluate our calculated power map we have randomly selected 20 cells (same $0.3 \mathrm{~m}$ cell side size) from the floor plan. At each of these cells we have made 50 power level measurements. For each cell, the average and the standard deviation of the 50 readings were calculated. Our measurements showed that the standard deviation of our readings (although being somewhat location dependent), are averaging to around $2 \mathrm{~dB}$, thus we can safely assume a $3 \mathrm{~dB}$ standard deviation for the measurements. We have calculated the square-root of the square mean of the difference between the calculated and measured values to be $1.65 \mathrm{~dB}$, thus our raytracing approach provides a sufficiently good estimate of the wireless propagation behavior in the sample house.

\section{B. Location Estimation Studies}

To evaluate the particle filtering-based location estimation approach, we have created a discrete event simulation program in $\mathrm{C}++$ that mimics the movement of a user inside the environment. Power level measurement samples are drawn according to the location of the user assuming a zero-mean Gaussian-noise model with a standard deviation of 3dB. Power reading samples were taken every half-second. To obtain the most likely position of the user we select the particle that has the most particles surrounding. We have defined a 190 second long movement path using pedestrian speed $(1 \mathrm{~m} / \mathrm{s})$ visiting several rooms of the house as depicted in Figure 4.

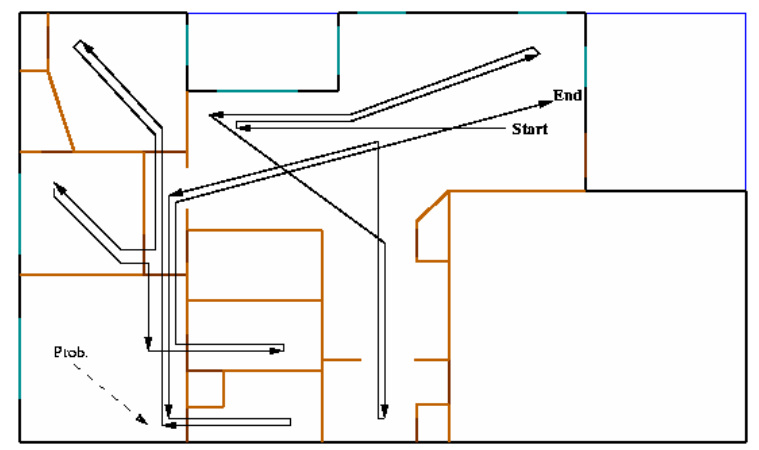

Figure 4. Movement path of user in the sample house.

To observe the behavior of the particles we have also created a graphical interface where the user, the particles, and the location estimate of the user are displayed after each sampling step. Figure 5 shows a short sequence of particle behavior $^{1}$. The user and the single point estimate are denoted

\footnotetext{
${ }^{1}$ Due to the limitations of paper a moving image of particles cannot be nicely presented.
}

with the letters $\boldsymbol{U}$ and $\boldsymbol{E}$ respectively, while the particles are represented with red (gray) dots or dot-clouds.

We have run simulations to evaluate the location estimates' precision as a function of the number of particles. We measured the estimates' precision by calculating the average Euclidian distance between the user and the location estimate.

Figure 6 shows the impact of the number of particles on the estimates' precision for all motion models. The wall considering and the 4D particle models' estimates come on average as close as 1 meter to the location of the user.

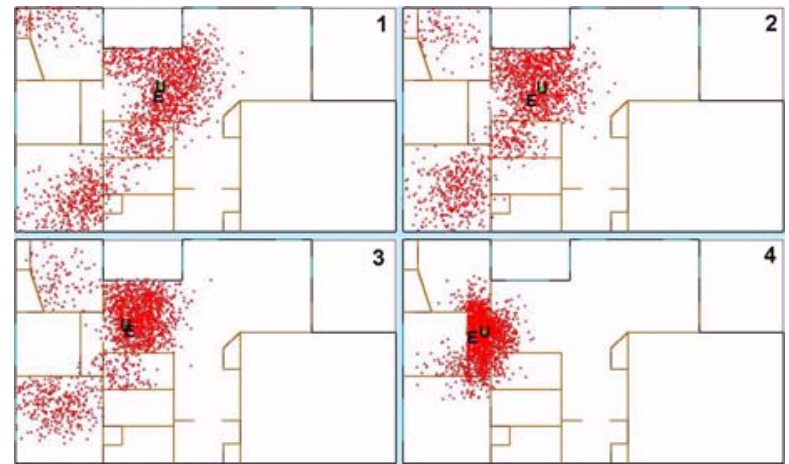

Figure 5. Particle filtering in progress $(\mathrm{U}=$ user; $\mathrm{E}=$ single location estimate).

To investigate how the location estimates’ precision varies over time for a fixed particle number, the deviations between actual and estimated location were recorded along the path of the user. Figures 7,8, and 9 show the corresponding graphs.

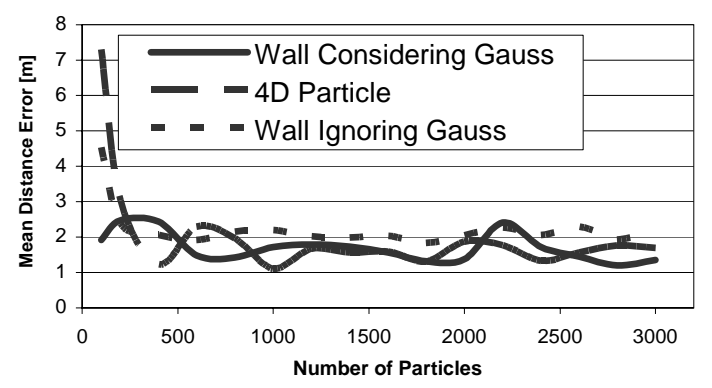

Figure 6. Number of particles vs. precision of the location estimate.

Although, the simple zero-mean velocity Gaussian system model performs the worst it still provides with an amazing performance. The wall considering and the 4D particle filter models perform similarly, with the former having less but longer error bursts. The latter two models rarely miss the room the user is at, thus we argue that our location system can be deployed in a smart environment to locate users.

\section{CONCLUSIONS}

In this paper we presented an approach for localization that uses existing RSSI measurements and a map of the expected wireless power measurements to estimate the position of a mobile node equipped with a wireless network card. To facilitate this we presented a multi-step technique that permits 
the construction of the wireless power map with a significantly reduced requirement for actual measurements. As opposed to previous approaches, the techniques introduced here do not require a human labor extensive model construction phase and can operate successfully with minimal infrastructure. To achieve the latter we use Monte Carlo filtering techniques to efficiently estimate the distribution of mobile node locations.

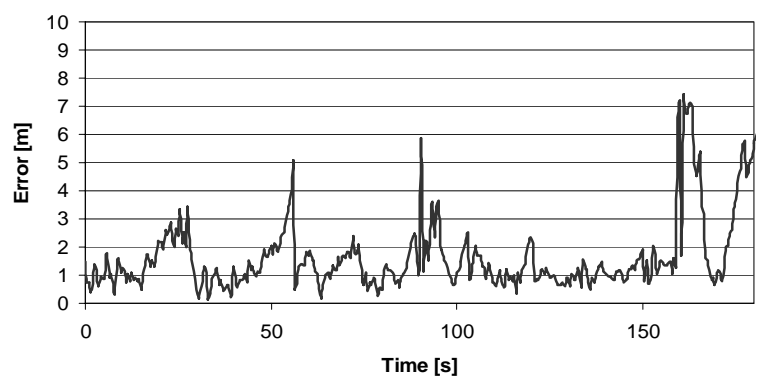

Figure 7. Wall ignoring model error vs. simulation time.

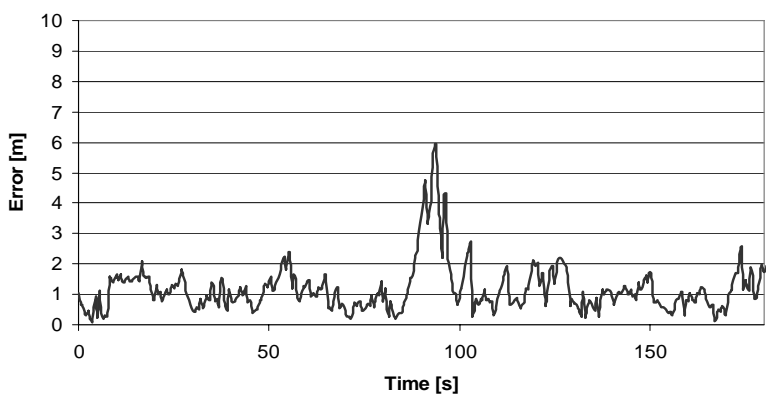

Figure 8. Wall considering model error vs. simulation time.

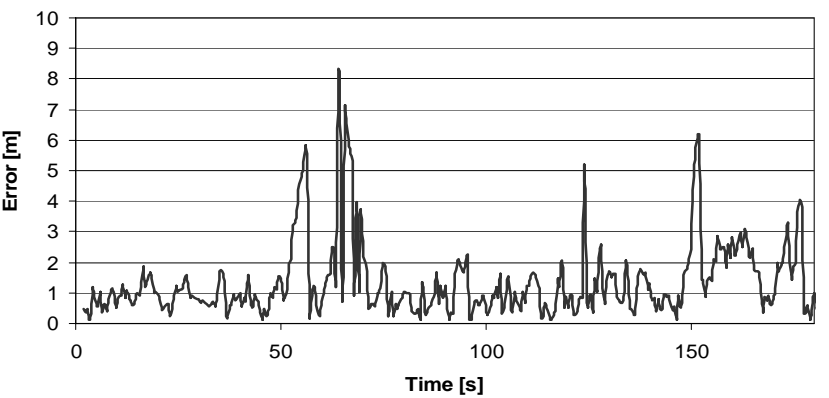

Figure 9. 4-D particle model error vs. simulation time.

To demonstrate the performance and applicability of the techniques, experiments were performed in a home environment. First, a wireless power map was constructed using the presented ray-tracing technique and compared to the actual readings in the home. This comparison showed that the constructed map is within approximately $2 \mathrm{~dB}$ of the actual measurements. Using this map, three movement models were implemented in the MC sampling-based location estimators and simulation studies were performed to evaluate the precision. The experiments showed that the filters were successful at estimating the mobile node's location. It has been demonstrated that even a simple movement model can produce results with an average precision around $1 \mathrm{~m}$. We consider showing that sub-room precision positioning is possible with RSSI readings from a single access point as our major contribution.

\section{REFERENCES}

$1 \quad$ P. Bahl, and V.N. Padmanabhan, "RADAR: An In-building RF-based User Location and Tracking System,” Proceedings of IEEE Infocom 2000, vol. 2, pp. 775-784, Tel Aviv, Israel, March 2000.

2 P.J. Brown, J.D. Bovey, and X. Chen, "Context-Aware Applications: From the Laboratory to the Marketplace,” IEEE Personal Communications Magazine, vol. 4, pp. 58-64, October 1997.

3 P. Castro, P. Chiu, T. Kremenek, and R. Muntz, "A Probabilistic Room Location Service for Wireless Networked Environments,” Ubiquitous Computing 2001, September 2001.

4 A. Doucet, N. de Freitas, and N. Gordon (editors), "Sequential Monte Carlo Methods in Practice," Springer-Verlag, 2001.

5 D. Fox, W. Burgard, F. Dellaert, and S. Thrun, "Monte Carlo Localization: Efficient Position Estimation for Mobile Robots”, National Conference on Artificial Intelligence, Orlando, FL, 1999.

$6 \quad$ A. Gelb (editor), “Applied Optimal Estimation,” MIT Press, 1974.

7 I.A. Getting, “The Global Positioning System,” IEEE Spectrum, vol. 30, pp. 36-47, December 1993.

8 M. Hassan-Ali, and K. Pahlavan, "Site-specific Wideband and Narrowband Modeling for Indoor Radio Channel Using Ray-tracing," Proceedings of the PMIRC’98, Boston, MA, 1998.

9 M. Hassan-Ali, and K. Pahlavan, "A New Statistical Model for SiteSpecific Indoor Radio Propagation Prediction Based on Geometric Optics and Geometric Probability,” IEEE Transactions on Wireless Communications, vol.1, no. 1, pp. 112-124, January 2002.

10 J. Hightower, and G. Borriello, "Location Systems for Ubiquitous Computing,” IEEE Computer Magazine (Special Issue on Location Aware Computing), vol. 34, no. 8, pp. 57-66, August 2001.

11 M. Isard and A. Blake, Contour Tracking by Stochastic Propagation of Conditional Density”, European Conference on Computer Vision, Cambridge, UK, pp. 343-356, 1996.

12 J. Krumm, S. Harris, B. Meyers, B. Brumitt, M. Hale, S. Shafer, "MultiCamera, Multi-Person Tracking for Easy Living,” IEEE International Workshop on Visual Surveillance, pp. 3-10, Piscataway, NJ, 2002.

13 A.M. Ladd, K. Bekris, A. Rudys, G. Marceau, L.E. Kavraki, and D.S. Wallach, "Robotics-Based Location Sensing Using Wireless Ethernet," ACM MobiCom, pp. 227-238, Atlanta, GA, September 2002.

14 T.Liu, P. Bahl, and I. Chlamtac, “A Hierarchical Position-Prediction Algorithm for Efficient Management of Resources in Cellular Networks,” IEEE GLOBECOM ’97, Phoenix, AZ, Nov. 1997.

15 R.J. Orr, and G.D. Abowd, "The Smart Floor: A Mechanism for Natural User Identification and Tracking," Conference on Human Factors in Computing Systems, pp. 1-6, Hague, Netherlands, April 2000.

16 N.B. Priyantha, A. Chakraborty, and H. Balakrishnan, "The Cricket Location-Support System,” ACM MobiCom, Boston, MA, August 2000.

17 T. Roos, P. Myllymaki, H. Tirri, P. Misikangas, and J. Sievanen, "A Probabilistic Approach to WLAN User Location Estimation,” Intnl Journal of Wless Information Nets, vol. 9, no. 3, pp. 155-164, July 2002.

18 S. Russel, P. Norvig, “Artificial Intelligence - A Modern Approach,” Prentice Hall, NJ, 1995.

19 A. Smailagic, D.P. Siewiorek, J. Anhalt, D. Kogan, and Y. Wang, "Location Sensing and Privacy in a Context-Aware Computing Environment,” IEEE Wireless Comm. Magazine, pp. 10-17, Oct.2002.

20 S. Tekinay (editor), IEEE Communications Magazine, Special Issue on Wireless Geolocation Systems and Services, April 1998.

21 S. Thrun, "Particle Filters in Robotics," Proceedings of the 17th Annual Conference on Uncertainty in AI (UAI), Edmonton, August 2002.

22 T. Tsukiyama, "Global Navigation System with RFID Tags," SPIE Mobile Robots Conference, pp. 256-264, Newton, MA, October 2001.

23 R. Want, A. Hopper, V. Falco, and J. Gibbons, "The Active Badge Location System,” ACM Transactions on Information Systems, vol. 10, no. 1, pp. 91-102, January 1992. 
24 M. Youssef, A. Agrawala, A.U. Shankar, and S.H. Noh, " A Probabilistic Clustering-Based Indoor Location Determination System,” Tech. Report, University of Maryland CS-TR 4350, March 2002. 